\title{
The physiology of pain: an update and review of clinical relevance
}

\author{
Dissanayake D W N ${ }^{1}$, Dissanayake D M D2 \\ Journal of the Ceylon College of Physicians, 2015, 46, 19-23
}

\section{Introduction}

Pain, defined as a symptom, sign or a syndrome, has been researched extensively compared to any other area in neurophysiology. This short review aims to explain the neurophysiological processes involved in the perception of pain, including some of the recent findings, which have led to novel targets of pain relief.

\section{Classification}

Pain is a complex perceptual experience. It has been classified based on pathophysiology, type of stimulus, site, duration, and severity. A recent, widely accepted pathophysiological classification classifies it as nociceptive, neuropathic and psychogenic. ${ }^{1,2}$ Sensation evoked as a result of localized stimulation of pain receptors (i.e. nociceptors) via mechanical, chemical or thermal modalities is referred to as nociceptive pain. Pain occurring as a result of abnormal signaling due to an injury or dysfunction of peripheral nociceptive neurons is classified as neuropathic pain. Psychogenic pain is the occurrence of pain sensation as a result of a psychiatric disorder in the absence of injury or inflammation in the affected site.

\section{Neuroanatomy}

Irrespective of its pathophysiology or categories, pain is commonly perceived as an unpleasant sensation. It is initiated by a noxious stimulus that generates impulses, which then ascend in pathways in the spinal cord to transmit information from the nociceptors to the brain.

The nerve endings containing the nociceptors are the primary afferents, terminating in the dorsal horn of the spinal cord. These nerves are small myelinated $A$ delta fibers and unmyelinated $C$ fibers. Following the stimulation of nociceptors, the generated action potential is propagated along these primary afferents, resulting in the release of excitatory amino acids (e.g.

\footnotetext{
${ }^{1}$ Department of Physiology, Faculty of Medicine, Colombo 08, Sri Lanka.

${ }^{2}$ Kingston Hospital NHS Trust, Galsworthy Road, Kingston Upon Thames, Surrey, KT2 7QB, UK.

Corresponding author: DWND,

E-mail: dilshani2003@yahoo.co.uk
}

glutamate and aspartate), neurotrophins (e.g. Brain Derived Neurotrophic Factor; BDNF) and peptides such as substance $P(S P)$, Neurokinin $A$ and Calcitonin Gene Related Peptide (CGRP) from the nerve endings in the dorsal horn of the spinal cord..$^{3,4,5}$

There are multiple neuronal networks involved in sensory-discriminative component (i.e. intensity, location, duration, temporal pattern etc.) and affectivecognitive component (i.e. relationship between pain with mood, memory, tolerance etc.) of pain sensation. Recent neuro-imaging techniques have been successful in defining some of the cortical regions involved in these multiple circuits. The somatosensory area of the post-central gyrus, ventroposterolateral and ventroposteromedial neuclei of the thalamus are found to play a major role in the sensory-discriminative component of pain..$^{5,6}$ The somatosensory area II in the lateral parietal cortex, the ventromedular and ventroposterioinferior nuclei of the thalamus, the inferior parietal cortex, the anterior cingulated cortex, the prefrontal cortex, the insular cortex, the amygdale and hippocampus play a part in the affective-cognitive component. ${ }^{5,6}$ Furthermore, the lateral spinothalamic tract, which is the ascending pathway in the spinal cord, contains discrete pathways to carry sensorydiscriminative (neospinothalamic) and cognitiveaffective (paleospinothalemic) components of pain. .,6,7,8 $^{5}$

The communication between the neurons of the pain pathways is mainly via chemical neurotransmitters. Several neurotransmitters and their receptors transmit and modulate the sensation of pain.

\section{Chemical transmission}

A painful stimulus causes inflammatory changes at the site of stimulation, resulting in activation of inflammatory mediators and immune cells. The immune cells produce cytokines and protons, which act on nociceptors to depolarize the cell membrane and generate action potentials, which can be transmitted along the nerve fibers of the pain pathway. The depolarization of the nociceptive membrane is due to the opening up of voltage and ligand-gated ion channels (e.g. $\mathrm{Na}^{+}, \mathrm{Ca}^{+2}, \mathrm{P} 2$, transient receptor potential-TRP). The sensitized immune cells also secrete nerve growth factors (NGF, BDNF), which alter the sensitivity of the nociceptors by acting on tyrosine kinase receptors. ${ }^{3,4,9}$ Lowering the threshold of the 
nociceptors leads to a prolonged response to stimulation. In the presence of nerve damage following injury, the degenerating nerves release CGRP and substance $P$, which enhance the production and release of more inflammatory mediators, thus leading to prolonged pain sensation. ${ }^{10}$ BDNF activates tyrosine kinase receptors $B$ in the secondary afferents in the spinal cord. ${ }^{5}$ Substance P (SP) acts on Neurokinin 2 receptors in the secondary afferents and contribute to the induction of dorsal horn sensitization. ${ }^{5,11}$ CGRP causes $\mathrm{Ca}^{2+}$-influx and prevents the metabolism of SP, and increases the release of SP and excitatory amino acids. Thus, CGRP strengthens the process of sensitization..$^{3,4}$ The excitatory amino acids, especially glutamate, bind with the receptors including ionotropic receptors such as AMPA (-amino-3-hydroxy-5-methyl4-isoxazolepropionic acid), NMDA (N-methyl-Dasparate), Kainate and metabotropic (acting via Gproteins to soluble second messengers) glutamate receptors in the presynaptic and postsynaptic secondorder neurons in the pain pathway..$^{10,12}$ The NMDA receptors are considered to play an important role in the transmission of chronic persistent pain and have been found responsible for the induction and initiation of hyperalgesia (i.e. increased pain sensation to a mild noxious stimulus) and allodynia (i.e. pain sensation to an innocuous stimulus). It has also been suggested that hyperalgesia and allodynia occur due to the rewiring in the dorsal horn, with abnormal sprouting of Abeta fibers which forms synapses with A-delta and $C$ fibres, bypassing the inhibitory interneurons and the descending modulatory pathways. ${ }^{13,14}$ Inhibitory interneurons secreting gamma amino butyric acid (GABA) modulate pain transmission at the superficial layers of the dorsal horn by presynaptic inhibiton of pain fibers, thus preventing the release of excitatory amino acids. In an injury, especially with neuronal damage, the GABAergic influence is diminished due to increased release of excitatory amino acids and also hyperactivation of NMDA receptors, which can cause apoptosis of inhibitory interneurons. ${ }^{15}$

In the presence of persistent inflammatory stimulation, changes occur in genetic regulation, which leads to induction of new proteins and modulation of the levels of expression of existing neurochemicals mediating pain. ${ }^{5,13}$ This transcription-dependent central sensitization may explain the persistent refractory pain syndromes which are sometimes independent of peripheral noxious stimulation and resistant to treatment.

\section{Modulatory pathways}

The human nervous system is equipped with innate inhibitory and excitatory mechanisms and pathways to modulate the sensation of pain. The majority of the mechanisms are inhibitory and are activated with the initiation of nociceptive information to alleviate pain. The descending pathways originating from cortical and sub-cortical areas modulate transmission of pain, mainly at the dorsal horn of the spinal cord.

The descending noradrenergic pathway from the locus coeruleus stimulates adrenergic receptors (mainly alpha-2) in the dorsal horn, resulting in a decrease in the release of substance $P$, hyperpolarizing the sensory afferents and depolarizing the GABAergic neurons to inhibit pain transmission. ${ }^{16,17}$ The descending serotonergic pathway from the nucleus raphe magnus has both inhibitory effects (by activating 5-HT1A and 5-HT7 receptors) and excitatory effects (via 5-HT2A and 5-HT3 receptors) on pain transmission. ${ }^{18,19}$

The opioid system is a major inhibitory system of nociception in the body, containing receptors for endogenous and exogenous peptides referred to as $\beta$ endorphins, enkephalins and the dynorphins. The receptors for these opioids are distributed in the afferents in the peripheral nerves, spinal cord and the brain. Up to seventeen classes of opioid receptors have been postulated, out of which three have long been confirmed to be functionally important, with identified genes responsible for their expression. ${ }^{20,21}$ These receptor classes are $\mu$-mu, $\delta$-delta and $\kappa$-kappa. Apart from the three major classes, receptors such as sigma, nociceptin and toll like receptor are also found to be involved in the analgesic effect and the side effects (e.g. tolerance and dependence) of opioids. ${ }^{20,21}$

The opioid peptides modulate nociceptive input in two ways: 1) block neurotransmitter release by inhibiting $\mathrm{Ca}^{2+}$ influx into the presynaptic terminal and/ or 2) open potassium channels, which hyperpolarizes neurons and inhibits spike activity. High densities of opiate receptors are found in periaqueductal gray (PAG), nucleus raphe magnus (NRM), and dorsal raphe (DR) in the rostral ventral medulla, caudate nucleus (CN), septal nucleus, hypothalamus, habenula, hippocampus and the dorsal horn of the spinal cord. ${ }^{21}$ Opioid synthesis and activity have been found to be increased following acupuncture and placebo induced pain relief. $22,23,24$

The cannabinoid system exerts an on-demand nociceptive effect, with the receptors distributed in the CNS and periphery. The primary antinociceptive target of the endocannabinoids (i.e. AEA, arachidonylethanolamide; anadamide,2-AG,2-arachidonylglycerol) and exogenous cannabis is the CB1 receptor found in abundance in PAG, RVM, dorsal horn and the periphery. ${ }^{22,23} \mathrm{CB} 1$ is a G-protein coupled pre-synaptic receptor, which exerts its effects via the inhibition of neurotransmitter release by blockade of voltage dependent calcium channels and activation of potassium channels. 22, 23, 24 Endocannabinoids are secreted by post-synaptic neurons and are metabolized 
by enzymes found in the post-synaptic (e.g. FAAH, Fatty Acid Amide Hydrolase) and presynaptic (MAGL, monoacylglycerol lipase) neurons. The reuptake of endocannabinoids to the post-synaptic and uptake in to the pre-synaptic membrane for metabolism are suggested to be by a specific endocannabinoid membrane transporter, which is yet to be cloned. ${ }^{24}$ The CB2 receptor, which is found in the peripheral tissues, is also found to be involved in the initiation of nociceptive transmission. ${ }^{24}$

\section{New targets for pain relief}

Traditional analgesics such as non-steroidal antiinflammatory drugs (NSAIDs) and opioid agonists (e.g. morphine) are ineffective in some pain disorders and pose problems with side-effects, thus mandating search for novel therapeutic agents with superior efficacy and safety. An important target is the secretion and metabolism of neurotransmitters such as serotonin, norephinephrine, neurokinin, GABA, glutamate and their receptors such as NMDA. The ion channels such as sodium channels, N-type calcium channels, TRP and $\mathrm{P} 2$ receptors are increasingly being involved in studies on pain relief. The pain modulatory systems including the endocannabinoids and the CB receptors, central opioid receptors, nerve growth factors and glial cell are also potential targets for effective pain relief.

Traditional tricyclic anti-depressants (such as amitriptyline and imipramine), which inhibit the uptake of serotonin and noradrenaline from the descending analgesic pathways, are effective in neuropathic pain. ${ }^{5,25}$ However, the positive effects of the above agents may be compromised by their side effects, due to the widespread systemic effects involving other receptors, such as the cholinergic and histaminergic, leading to cardiorespiratory effects, glaucoma and urinary retention. ${ }^{25}$ Newer agents such as venlafaxine and duloxetine enhance both serotonin and norepinephrine activity in the pain modulatory pathways, with less affinity for cholinergic and histaminergic receptors. ${ }^{5,26}$ Both venlafaxine and duloxetine have been found to be effective in treating painful polyneuropathies and diabetic neuropathy, with less side effects. ${ }^{26,27}$

Anticonvulsants such as carbamazepine, sodium valproate, oxcarbazepine, topiramate, vigabatrin and levetiracetam have been found to demonstrate analgesic effects by enhancing GABA activity, inhibiting glutamate release, blocking NMDA receptors and blocking neuronal membrane $\mathrm{Ca}^{+2}$ and $\mathrm{Na}^{+}$channels. ${ }^{5}$ Carbamazepine and oxcarbazepine are effective analgesics in trigeminal neuralgia. However, the use of newer anticonvulsants has still not being proven to have superior efficacy compared to traditional analgesics in treatment of neuropathic pain or any other pain syndrome.
NMDA receptor antagonists such as dextromethorphan, amantadine, memantine and ketamine and compounds with varying degree of NMDA antagonism have been demonstrated to be effective analgesics. ${ }^{12,28,29}$ Such antagonists when combined with other effects such as central opioid effect (e.g. methadone), GABAergic effects (e.g., anti-convulsants) have shown varying degrees of analgesic properties and have shown high levels of efficacy in treating neuropathic pain and associated hyperalgesia and allodynia. ${ }^{5}$ But NMDA antagonism leads to unwanted psychological side-effects, more severe with systemic administration, which requires further research into an NMDA antagonist with high efficacy and less side effects.

Using compounds that directly target the ion channels in the peripheral and central nociceptive pathways is considered to have the potential for higher therapeutic efficacy with fewer side effects. TRPV1 antagonists such as SB-705498, AMG517, AMG628 and $A B T 102$ have all been found to be effective in the treatment of inflammatory and neuropathic pain in animal models. ${ }^{2,30,31,32}$ Since the TRPV1 modulators affect both central and peripheral pathways, further studies would be needed to identify potential side effects (e.g. hyperthermia) prior to translational studies.

Gabapentin and a more recent analogue pregabalin are widely used as treatment for neuropathic pain. The above analgesics, earlier considered as GABA analogues, are now found to be more specifically binding with voltage-gated calcium channels and inhibiting the release of glutamate, both at presynaptic and post synaptic sites, peripherally and centrally. ${ }^{5,33}$ Combination of opioids with gabapentin has demonstrated synergistic effects in relieving neuropathic pain. ${ }^{33}$

The endocannabinoid system has long been explored as an important modulatory pathway for pain relief. This system is postulated to be involved in the analgesic effects produced by some of the commonly used analgesics, including paracetamol. ${ }^{34}$ CB1 receptor agonists (e.g. THC, cannabidiol) have demonstrated efficacy in pain relief in chronic pain disorders. ${ }^{24,35}$ However, their use is greatly hampered by the unwanted psychotic effects. ${ }^{23,24,35} \mathrm{CB} 2$ receptor agonists have recently been shown to be effective in pain relief, possibly via its peripheral stimulatory activity on immune cells. ${ }^{36}$ Inhibitors of the metabolizing enzymes (e.g. FAAH and MAGL inhibitors) have demonstrated efficacy in reducing pain sensitivity in animal models of acute and chronic pain. ${ }^{37,38}$ The endocannabinoid system, especially with receptor and enzyme targets in the periphery, is an attractive target for the development of future analgesics.

Activated glial cells have been demonstrated to 
release pro-inflammatory mediators that contribute to neuropathic pain. Some potential targets for neuropathic pain include blocking glial cell activation, prevention of bio-synthesis of the cytokines and antagonize the action of the pro-inflammatory cytokines. ${ }^{2,31}$ Several animal studies have demonstrated the effectiveness of substances such as etanercept and anakinra in blocking glial cell-mediated cytokine signaling. ${ }^{2,31}$ The glial cells are emerging as an attractive target for treatment of chronic pain; this, however, needs a careful approach, considering the neuroprotective functions of these cells.

Adenosine triphosphate (ATP) induces pain by activating $P 1$ and $P 2$ receptors. The $P 2$ receptor, which is $G$ protein-coupled, has received attention as a potential target for the relief of acute and chronic pain. ${ }^{2}$ Compounds such as 1-benzyl-5-phenyltetrazoles and $\mathrm{N}$-acyl hydrazides have demonstrated their efficacy as antagonists to the $\mathrm{P} 2$ receptor sub-type $\mathrm{P} 2 \times 7 .{ }^{40}$ The $\mathrm{P} 2$ receptor is suggested as a very effective potential target for pain relief, with the recently developed small molecular antagonists demonstrating site-specific activity. However, further studies are needed to elucidate the side effects and adverse effects of these compounds.

\section{Conclusion}

The neurobiology of pain is continuously being explored and the novel therapeutic targets for pain relief are being tested. The multiple complex circuitries of the pain pathway, with central and peripheral modulation of the perception, make it a very complex sensation with sensory, cognitive and emotional components associated with it. The variety of neurochemicals and receptors involved in the perception of pain has led to the search for many targets of pain relief. Novel targets are being studied to counteract pain syndromes which are resistant to current treatment modalities. Site-specific and selective agents are receiving a lot of attention with the hope for high efficacy and minimal side effects.

\section{References}

1. Melnikova I. Pain market. Nature Reviews Drug Discovery 2010; 9: 589-90.

2. Rao PPN, Mohamed T. Current and emerging at-site pain medications: a review. Journal of Pain Research 2011; 4: 279-86.

3. Millan MJ. The induction of pain, an integrative review. Progress in Neurobiology 1999; 57: 1-164.

4. Willis WD, Westlund KN. Neuroanatomy of the pain system and of the pathways that modulate pain. Journal of Clinical Neurophysiology 1997; 14: 2-31.

5. Vranken JH. Elucidation of Pathophysiology and Treatment of Neuropathic Pain. Central Nervous System Agents in Medicinal Chemistry 2012; 12: 304-14.
6. Borsook D, Becerra L. How close are we in utilizing functional neuroimaging in routine clinical diagnosis of neuropathic pain? Current Pain Headache Reports 2011; 15: 223-29.

7. Brooks J, Tracey I. From nociception to pain perception, imaging the spinal and supraspinal pathways. Journal of Anatomy 2005; 207:19-33.

8. Price DD. Central neural mechanisms that interrelate sensory and affective dimensions of pain. Molecular Interventions 2002; 2: 392-402.

9. Aggugia M. Neurophysiology of Pain (review). Neurological Sciences 2003; 24: S57-60.

10. Costigan M, Scholz J, Woolf CJ. Neuropathic Pain: A Maladaptive Response of the Nervous System to Damage. Annual Review of Neuroscience 2009; 32: 1-32.

11. Woolf CJ, Shortland P, Reynolds M, Ridings J, Doubell T, Coggeshall RE. Reorganization of central terminals of myelinated primary afferents in the rat dorsal horn following peripheral axotomy. Journal of Comparative Neurology 1995; 360: 121-34.

12. Bennett GJ. NMDA-Receptor Antagonists: Evolving Role in Analgesia, Update on the Neurophysiology of Pain Transmission and Modulation: Focus on the NMDA-Receptor. Journal of Pain and Symptom Management 2000; 19: S2-6 (Proceedings Supplement).

13. Woolf $\mathrm{CJ}$. Central sensitization, implications for the diagnosis and treatment of pain. Pain 2011; 152: S2-S15.

14. Coull JA, Beggs S, Boudreau D, Boivin D, Tsuda M, Inoue K, Gravel C, Salter MW, De Koninck, Y. BDNF from microglia causes the shift in neuronal anion gradient underlying neuropathic pain. Nature 2005; 438: 1017-21.

15. Gwak YS, Hulsebosch CE. GABA and central neuropathic pain following spinal cord injury. Neuropharmacology 2011; 60: 799-808.

16. Budai D,Harasawa I, Fields HL. Midbrain periaq-ueductal gray (PAG) inhibits nociceptive inputs to sacral dorsal horn nociceptive neurons through alpha2-adrenergic receptors. Journal of Neurophysiology 1998; 80: 2244-54.

17. Gassner M, Ruscheweyh R, Sandkuhler J. Direct excitation of spinal GABAergic interneurons by noradrenalin. Pain 2009; 145: 204-10.

18. Doly S, Fischer J, Brisorgueil MJ, Verge D, Con-rath M. Preand postsynaptic localization of the $5-\mathrm{HT} 7$ receptor in rat dorsal spinal cord: immunocytochemical evidence. Journal of Comparative Neurology 2005 ; 490: 256-69.

19. Bardin L. The complex role of serotonin and 5-HT receptors in chronic pain. Behavioral Pharmacology 2011; 22: 390-404.

20. Díaz JL, Zamanillo D, Corbera J, Baeyens JM, Maldonado R, Pericàs MA, Vela JM, Torrens A. Selective sigma-1 (sigma1) receptor antagonists: emerging target for the treatment of neuropathic pain. Central Nervous System Agents in Medicinal Chemistry 2009; 9: 172-83.

21. Ossipov MH, Gregory OD, Porreca F. Central modulation of pain. The journal of clinical investigation 2010; 11: 3779-89.

22. Guindon J, Beaulieu P, Hohmann AG. Pharmacology of the cannabinoid system. In: Beaulieu P, Lussier D, Porreca F, Dickenson AH, editors. Pharmacology of pain. Seattle:IASP Press, 2010; 6. 
23. Pertwee RG. Pharmacological actions of cannabinoids. Handbook of Experimental Pharmacology 2005; 168: 1-51.

24. Ulugö A. The Endocannabinoid System as a Potential Therapeutic Target for Pain Modulation. Balkan Medical Journal 2014; 31: 115-20.

25. Swenson JR, Doucette S, Fergusson D. Adverse cardiovascular events in antidepressant trials involving highrisk patients, a systematic review of randomized trials. Canadian Journal of Psychiatry 2006; 51: 923-9.

26. Lunn MP, Hughes RA, Wiffen PJ. Duloxetine for treating painful neuropathy or chronic pain. Cochrane Database Systematic Review 2009; 7, 4,CD007115.

27. Vranken $\mathrm{JH}$, Hollmann $\mathrm{MW}$, van der Vegt $\mathrm{MH}, \mathrm{Kruis} \mathrm{MR}$, Heesen M, Vos K, Pijl AJ, Dijkgraaf MGW. Duloxetine in patients with central neuropathic pain caused by spinal cord injury or stroke, A randomized, double-blind, placebocontrolled trial. Pain 2011; 152: 267-3.

28. Carlsson KC, Hoem NO, Moberg ER, Mathisen LC. Analgesic effect of dextromethorphan in neuropathic pain. Acta Anaesthesiologica Scandandinavia 2004; 48: 328-36.

29. Eisenberg E, Pud D, Koltun L, Loven D. Effect of early administration of the $\mathrm{N}$-methyl-d-aspartate receptor antagonist amantadine on the development of postmastectomy pain syndrome, a prospective pilot study. Journal of Pain 2007; 8: 223-9.

30. Rami HK, Thompson M, Stemp G, Fell S, Jerman JC, Stevens AJ, Smart D, Sargent B, Sanderson D, Randall AD. Discovery of SB-705498: A potent, selective and orally bioavailable TRPV1 antagonist suitable for clinical development. Bioorganic and Medical Chemistry Letters 2006; 16: 3287-91.

31. Doherty EM, Fotsch C, BannonAW, Bo Y, Chen N, Dominguez C, Falsey J, Gavva NR, Katon J, Nixey T, Ognyanov VI, Pettus L, Rzasa RM, Stec M, Surapaneni S, Tamir R, Zhu J, Treanor JJ, Norman MH. Novel vanilloid receptor-1 antagonists: 2 . Structure-activity relationship of 4oxopyrimidines leading to the selection of a clinical candidate. Journal of Medical Chemistry 2007; 50: 3515-27.

32. Dai $Y$, Wang $S$, Tominaga M, et al. Sensitization of TRPA1 by
PAR2 contributes to the sensation of inflammatory pain. Journal of Clinical Investigation 2007; 117: 1979-87.

33. Gilron I, Bailey JM, Tu D, Holden RR, Weaver DF, Houlden RL. Morphine, gabapentin, or their combination for neuropathic pain. New England Journal of Medicine 2005; 352 : 1324-34.

34. Mallet C, Daulhac L, Bonnefont J, Ledent C, Etienne M, Chapuy E, Libert F, Eschalier A. Endocannabinoid and serotonergic systems are needed for acetaminophen-induced analgesia. Pain 2008; 139: 190-200.

35. Pertwee RG. Targeting the endocannabinoid system with cannabinoid receptor agonists: pharmacological strategies and therapeutic possibilities. Philosophical Transactions of the Royal Society of London B Biological Sciences 2012; 367: 3353-63.

36. Hsieh GC, Pai M, Chandran P, Hooker BA, Zhu CZ, Salyers AK, Wensink EJ, Zhan C, Carroll WA, Dart MJ, Yao BB, Honore P, Meyer MD. Central and peripheral sites of action for $\mathrm{CB} 2$ receptor mediated analgesic activity in chronic inflammatory and neuropathic pain models in rats. British Journal of Pharmacology 2011; 162: 428-40.

37. Naidu PS, Kinsey SG, Guo TL, Cravatt BF, Lichtman AH. Regulation of inflammatory pain by inhibition of fatty acid amide hydrolase. Journal of Pharmacology and Experimental Therapeutics 2010; 334: 182-90.

38. Schlosburg JE, Blankman JL, Long JZ, Nomura DK, Pan B, Kinsey SG, Nguyen PT, Ramesh D, Booker L, Burston JJ, Thomas EA, Selley DE, Sim-Selley LJ, Liu QS, Lichtman AH, Cravatt BF. Chronic monoacylglycerol lipase blockade causes functional antagonism of the endocannabinoid system. Nature Neuroscience 2010;13: 1113-U111.

39. Vallejo R, Tilley DM, Vogel L, Benyamin R. The role of glia and the immune system in the development and maintenance of neuropathic pain. Pain Practice 2010; 10: 167-171.

40. Nelson DW, Sarris K, Kalvin DM, Namovic MT, Grayson G, Donnelly-Roberts DL, Harris R, Honore P, Jarvis MF, Faltynek $\mathrm{CR}$, Carroll WA. Structure-activity relationship studies on $\mathrm{N}^{\prime}$-aryl carbohydrazide $\mathrm{P} 2 \mathrm{X} 7$ antagonists. Journal of Medicinal Chemistry 2008; 51: 3030-34. 